\title{
Characterization of Causal Agents of a Novel Disease Inducing Brown-Black Spots on Tender Tea Leaves in China
}

Z. H. Wang, Z. X. Zhao and N. Hong, State Key Laboratory of Agricultural Microbiology, Wuhan, Hubei 430070, P. R. China; Key Laboratory of Plant Pathology of Hubei Province, Wuhan, Hubei 430070, P. R. China; College of Plant Science and Technology, Huazhong Agricultural University, Wuhan, Hubei 430070, P. R. China; Dejiang Ni, College of Horticulture and Forestry Sciences, Huazhong Agricultural University, Wuhan, Hubei 430070, China; and L. Cai, ${ }^{\dagger}$ W. X. Xu, ${ }^{\dagger}$ and Y. N. Xiao, State Key Laboratory of Agricultural Microbiology, Wuhan, Hubei 430070, P. R. China; Key Laboratory of Plant Pathology of Hubei Province, Wuhan, Hubei 430070, P. R. China; College of Plant Science and Technology, Huazhong Agricultural University, Wuhan, Hubei 430070, P. R. China

\begin{abstract}
A novel disease characterized by small brown-black spots ( 1 to $2 \mathrm{~mm}$ in diameter) on tender tea leaves (Camellia sinensis) has been observed in many regions of Hubei Province, China, which severely affects the yield and quality of tea. Tea leaf samples with typical symptoms were collected from three major tea-cultivation regions of Hubei, and were subjected to pathogen isolation for etiological analysis. As a result, 34 Pestalotiopsis isolates were obtained from 20 samples, and they were identified as Pestalotiopsis theae (14 isolates), P. camelliae (12), and $P$. clavispora (8), determined by morphologies and phylogenetic analysis based on internal transcribed spacer, and partial $\beta$-tubulin and translation elongation factor 1-alpha genes. Pathogenicity tests on detached tea leaves showed that no matter what mycelial discs or conidium suspensions were used, inoculation of the Pestalotiopsis fungi could result in small brown-black spots ( 1 to $2 \mathrm{~mm}$ in diameter) on wounded leaves, similar

to those observed in the field in the sizes and colors. It also revealed that only $P$. theae had pathogenicity on unwounded tea leaves, and $P$. theae and $P$. clavispora showed significantly higher virulence than $P$. camelliae. Inoculation test with conidium suspension on intact tea leaves in the field further confirmed that $P$. theae as the pathogen of brownblack spots. Reisolation of the pathogens from diseased leaves confirmed that the symptom was caused by the inoculation of Pestalotiopsis fungi. The $P$. theae isolates responsible for brown-black spots were also compared with those for tea gray blight disease in growth rate, pathogenicity, and molecular characteristics in parallel. To our knowledge, this is the first report that the Pestalotiopsis fungi cause brown-black spot disease on tender tea leaves. The results provide important implications for the prevention and management of this economically important disease.
\end{abstract}

Tea is one of the three main nonalcoholic beverages (tea, coffee, and cocoa) worldwide, and is the most widely consumed drink after water in the world (Bond 2012). Tea contains some 4,000 bioactive compounds (Mahmood et al. 2010), many of which are beneficial for human health, such as polyphenols, caffeine, vitamins, amino acids, proteins, polysaccharides, and trace elements, which are helpful for the prevention and treatment of cancer, cardiovascular and cerebrovascular diseases, and improvement of immunity (Bhatt et al. 2010; Namita et al. 2012). Tea (Camellia sinensis [L.] O. Kuntze) originated from southwestern China and is currently planted in more than 1,000 counties in 20 provinces of China (Z. M. Chen and X. L. Sun 2013). For a long time, tea yield in China has been ranked first in the world, and tea is still crucial in local economies and people's daily life (Ma et al. 2014).

However, diseases pose serious threats to tea production around the world. More than 100 diseases have been found in China, about 15 of which occur on buds and tender leaves (Z. M. Chen and X. L. Sun 2013). Among these diseases, blister blight, net blister blight, white scab, bud blight, leaf blight, gray blight, and brown round spot can be epidemic and/or affect the quality of the product (Z. M. Chen and X. L. Sun 2013). In recent years, a novel disease characterized by small brown-black spots (1 to $2 \mathrm{~mm}$ in diameter) has been observed in many tea gardens of Hubei Province, China. The disease poses a big potential threat for tea production in Hubei, since many varieties susceptible to this disease are widely cultivated, e.g., C. sinensis var.

${ }^{\dagger}$ Corresponding authors. Wenxing Xu, xuwenxing@mail.hzau.edu.cn; and Li Cai, caili@mail.hzau.edu.cn

*The $\boldsymbol{e}$-Xtra logo stands for "electronic extra" and indicates that three supplementary figures are published online.

Accepted for publication 31 May 2017.

() 2017 The American Phytopathological Society
Enshitaizicha, susceptible to this disease, is widely cultivated in Enshi prefecture (over 60,000 ha).

Although several tea leaf diseases have been reported in China, only white scab and brown round spot diseases can cause brownblack spots on tender leaves (Z. M. Chen and X. L. Sun 2013; Yang et al. 2012). Typically, the spots caused by white scab have brown edge and a large grayish white center, and brown round spot causes wholly brown-black spots with a small grayish white center (Z. M. Chen and X. L. Sun 2013). For the disease in the present study, the spots are wholly dark brown without any white part in the center. Hence, this disease might be a novel tea leaf disease and is here designated as tea leaf brown-black spot disease. Due to the economic importance of tea in China, it is imperative to develop proper strategies to prevent or mitigate the impact of this new disease. For this purpose, we have investigated for the first time the etiology of this novel disease.

\section{Materials and Methods}

Collection of samples. In July and August of 2013, 20 leaf samples showing typical brown-black spots 1 to $2 \mathrm{~mm}$ in diameter were collected from Xuan'en, Yichang and Yingshan counties, Hubei Province, China. Simultaneously, 12 leaf samples showing the typical symptoms of tea gray blight disease were collected from Yichang, Yingshan, and Zhuxi counties, Hubei Province, and kept in a refrigeration house at $4^{\circ} \mathrm{C}$ until use.

Isolation of fungi. For leaf samples showing brown-black spots, four to five leaf blocks ( 4 to $5 \mathrm{~mm}^{2}$ ) containing at least one lesion were excised from each diseased leaf after they were surface-sterilized with $0.1 \% \mathrm{HgCl}_{2}$ for $1 \mathrm{~min}$ and $75 \%$ ethanol for $1 \mathrm{~min}$, rinsed twice with sterilized water, and cultured on potato dextrose agar (PDA, 20\% diced potatoes, $2 \%$ glucose, and $1.5 \%$ agar) plates for fungal colony formulation as previously reported (Bai et al. 2015). For leaf samples showing gray blight disease symptoms, pathogen isolation was conducted by culturing the conidia picked from acervuli over the lesions. The resulting colonies were further purified by culturing a single conidium according to the previous report (Bai et al. 2015). The isolates that did not sporulate 
were purified by the single-mycelium tipping method (Yaegashi et al. 2012).

Morphology characterization of fungi. Mycelial discs taken from active growth areas were inoculated on PDA plates and incubated in the dark at $25^{\circ} \mathrm{C}$ for 4 days. Typical colony morphology, mycelial density, and aerial hypha growth were observed under a microscope (Nikon Eclipse E600 FN, Nikon, Japan). Fifty conidia of each isolate were measured and photographed with SPOT 4.6 (Diagnostic Instruments, Inc., U.S.A.). Morphological characterization of the fungi was conducted according to the standards described by Ge et al. (2009) and Guba (1961).

Determination of growth rate. PDA discs ( $5 \mathrm{~mm}$ in diameter) covered with mycelia were taken from the edge of a growing colony with a sterile puncher. One disc was placed in the center of PDA plate and incubated in the dark at $25^{\circ} \mathrm{C}$. The colony diameter was measured using the cross intersect method and recorded daily during 4 days of incubation. The growth rate was calculated with the subtraction of the diameter of initial disc. The experiment was performed in three replicates for each isolate.

Molecular characterization of fungi. Genomic DNA of fungi was extracted according to the cetyltrimethylammonium bromide (CTAB) method as previously described (Freeman et al. 1996). The extracted DNA was subjected to a amplification in a PCR Thermal Cycler (Model PTC-100, MJ Research, U.S.A.) of three regions including the ribosomal internal transcribed spacer (ITS, including a partial 18S ribosomal RNA gene, internal transcribed spacer $1,5.8 \mathrm{~S}$ ribosomal RNA gene, internal transcribed spacer 2, and partial $28 \mathrm{~S}$ ribosomal RNA gene), the partial $\beta$-tubulin (TUB), and partial translation elongation factor 1-alpha $(T E F)$ genes using the primer pairs ITS1/ITS4 (5'-TCCGTA GGTGAACCTGCGG-3'/5'-TCCTCCGCTTATTGATATGC-3') (White et al. 1990), and $\mathrm{Bt} 2 \mathrm{a} / \mathrm{Bt} 2 \mathrm{~b} 5^{\prime}$-GGTAACCAAATCGGTGCTGCT TTC-3'/5'-ACCCTCAGTGTAGTGACCCTTGGC-3') (Glass and Donaldson 1995; O'Donnell and Cigelnik 1997), and EF1-728F/ EF-2 (5'-CATCGAGAAGTTCGAGAAGG-3'/5'-TACTTGAAGG AACCCTTACC-3') (Carbone and Kohn 1999; O'Donnell et al. 1998), respectively. Amplifying conditions were programmed as predenaturing at $94^{\circ} \mathrm{C}$ for $4 \mathrm{~min}$, followed by 35 cycles of denaturing at $94^{\circ} \mathrm{C}$ for $30 \mathrm{~s}$, annealing at $54^{\circ} \mathrm{C}$ for $30 \mathrm{~s}$, extension at $72^{\circ} \mathrm{C}$ for $1 \mathrm{~min}$, and finally complementary extension at $72^{\circ} \mathrm{C}$ for $7 \mathrm{~min}$. The PCR products were detected in agarose gel electrophoresis (1\%), and the target DNA bands were recovered using DNA gel extraction kit (Wuhan More Biotechnology Corporation, Wuhan, China), and subjected to sequencing by Sangon Biotech (Shanghai) Co., Ltd, China.

The sequences were compared with the best matches available in GenBank with BLASTn (http://blast.ncbi.nlm.nih.gov). Multiple alignments of DNA sequences was conducted using MAFFT 6.85 (http://mafft.cbrc.jp/alignment/software/) with the default settings. DNA sequences were edited and annotated with GeneDoc (Nicholas et al. 1997; http://www.nrbsc.org/gfx/genedoc/ebinet.htm). A phylogenetic tree was constructed using MEGA 5.2.2 (Tamura et al. 2011; http://megasoftware.net/) with neighbor-joining (NJ) or maximum likelihood (ML) method, with the default parameters and bootstrap values of 1,000 replicates.

Pathogenicity analysis. Pathogenicity analysis was conducted according to a previous reports (Wang et al. 2016; Zhou and $\mathrm{Xu}$ 2014). Tender leaves were collected from $C$. sinensis var. Taicha No. 12. Before inoculation, leaves were disinfected in alcohol (75\%), rinsed with sterile water several times, and air-dried. Colonized agar discs were prepared from fresh colony as described above and put on the upside of detached tea leaves that were wounded six times with a needle (insect pin, $0.45 \mathrm{~mm}$ in diameter) or left unwounded. Uncolonized discs were used as controls. For inoculation of conidia, $50 \mu \mathrm{l}$ of a suspension at the concentration of $10^{5} \mathrm{conidia} / \mathrm{ml}$ was inoculated onto the upside of each tea leaf (over the six needle-piercing holes) and covered with sterilized cotton on wounded or unwounded leaves. Control leaves were inoculated in parallel using sterile water. The inoculated leaves were incubated in a climate chamber at $25^{\circ} \mathrm{C}$ under $12 \mathrm{~h}$ of intermittent light. Diameter of disease spot was measured 3 days after inoculation. The experiment was performed in four replicates for each treatment.
Intact leaves inoculations were performed on newly developed leaves on tea trees (C. sinensis var. Enshitaizicha) that were cultivated in Wujiatai, Xuan'en County, Enshi, Hubei Province, China, from August to September of 2016. A $100 \mathrm{ml}$ aliquot of spore suspension $\left(10^{5}\right.$ conidia/ml $)$ was sprayed onto approximately 20 leaves. Potato dextrose (PD, 20\% diced potatoes and 2\% glucose) solutions containing no conidia were used in parallel as controls. Three replicates were performed for the inoculation. The symptoms were monitored and recorded every 10 days until the experiment was complete.

Data statistics and analysis. Data were analyzed with SPSS Statistics 21.0 (WinWrap Basic; http://www.winwrap.com) by one-way ANOVA, and means were compared using Tukey's test at a significance level of $P=0.05$. Homogeneity of variance was tested before analysis.

\section{Results}

Field symptom and loss survey. This disease occurs on the tender leaves of many tea varieties, including Fuyun No. 6, Fuyun No. 1, Taicha No. 2, and Enshitaizicha, in Enshi prefecture, Yichang and Huanggang, Hubei Province, China. In the early stage, pinpoint-sized and light yellow spots are observed on the surface of infected leaves, and part of the spots are surrounded by yellowish or reddish-brown halos; in the late stage, the spots gradually turn blackish or reddish brown, and the spot sizes grow up to 1 to $2 \mathrm{~mm}$ in diameter (Fig. 1A and B). During the outbreak of this disease, numerous spots can be formed on a single leaf and finally fuse into a large lesion, resulting in a necrosis on the leaf. A survey in Ma'an village of Xuan'en county, Enshi prefecture, Hubei, revealed that this disease breaks out annually and the affected area is usually over 20 ha, leading to a $50 \%$ economic loss. Additionally, this disease causes apparent deterioration of tea quality, as the prepared tea is very brittle and tastes bitter and astringent, like raw persimmon.

Culture properties of the isolates from tea leaf samples showing typical brown-black spots. Twenty leaf samples showing typical brown-black spots (round, sunken, and 1 to $2 \mathrm{~mm}$ in diameter) (Fig. 1A and B) were collected for pathogen isolation, and a total of 44 purified fungal isolates were obtained, among which 34 showed typical colony characteristics of Pestalotiopsis spp. from the collected samples. All colonies were wholly white at the beginning of incubation and were classified into three different groups (I to III) at the end of incubation (Fig. 2A).

Group I contained 14 isolates (Table 1) such as TP1-2O and TP2$2 \mathrm{~W}$. Mycelia were white in the early stage and turned grayish white later. Typically, mycelia were flocculence-like and formed three to four concentric aerial rings in each colony. No pigments were produced (Fig. 2A, group I).

Group II included 12 isolates (Table 1) such as the representative LT3-2O, LT4-1O, and LT4-2B. Mycelia were originally snow-white, and then turned grayish white in the late stage, producing light- to yellow-brown pigments on the undersides of the colonies. Mycelia grew along the agar surface, and the aerial hyphae were sparse (Fig. 2A, group II).

Group III included the final 18 isolates (Table 1), such as TP2-1A and PF2-8O. Mycelia were also white in early growth and later changed to grayish white and flocculence-like appearance, and no pigments were produced, similar to the isolates of group I. Noticeably, concentric aerial hyphal rings could also be formed, while the rings were less apparent and the number was smaller than that of group I (Fig. 2A, group III).

Microscopic characteristics of the Pestalotiopsis isolates. For isolates in group I, the conidia were fusoid, straight, or a bit curved. Each conidium had five cells, among which the three median cells were dark brown, and the two end cells were hyaline. Conidia had lengths of 20.1 to $30.9 \mu \mathrm{m}$ and widths of 3.7 to $8.3 \mu \mathrm{m}$. Each conidium formed two to four apical appendages and one basal appendage. The apical and basal appendages had lengths of 19.22 to $41.41 \mu \mathrm{m}$ and 3.6 to $8.7 \mu \mathrm{m}$, respectively (Table 2, Fig. $2 \mathrm{~B}$, group I).

For the isolates in group II, except for LT3-2O, which could not produce conidia under the tested conditions, the conidia were also fusoid. Each conidium contained five cells, among which the top one was hyaline, the middle three were light brown, and the basal 
one was a bit paler than the brown cells. Conidia showed lengths of 28.8 to $34.3 \mu \mathrm{m}$ and widths of 5.6 to $8.8 \mu \mathrm{m}$. Each conidium formed two to five but mainly three to four apical appendages with lengths of 15.5 to $38.7 \mu \mathrm{m}$, while no basal appendage was formed (Table 2, Fig. 2B, group II).

For the isolates in group III, the fusoid conidia also had five cells, among which the first two median cells were dark brown, the third median cell was light brown, and the two end cells were hyaline. Conidia were straight or a bit curved, 19.8 to $26.3 \mu \mathrm{m}$ in length and 4.9 to $8.7 \mu \mathrm{m}$ in width. Each conidium produced two to four apical appendages and one basal appendage. The apical and basal appendages had lengths of 15.2 to $29.3 \mu \mathrm{m}$ and 4.1 to $9.3 \mu \mathrm{m}$, respectively (Table 2, Fig. 2B, group III).

The group I, II, and III isolates were tentatively identified as $P$. theae, $P$. camelliae, and $P$. clavispora according to their colony and microscopic characteristics, respectively, in comparison with type species (Table 2). Some representative isolates (TP1-2O and TP2-2W from group I, LT4-1O and LT4-2B from group II, and TP2-1A and PF2$8 \mathrm{O}$ from group III) were used for most further analyses.
Growth rate of the representative Pestalotiopsis isolates. The isolates showed various growth rates that displayed significant differences even within the same group (Fig. 3A). LT3-2O of $P$. camellia grew at a rate of only $5.6 \mathrm{~mm} /$ day, which was the lowest, while TP1$2 \mathrm{O}$ (P. theae) and TP-2-1A (P. clavispora) had the same rate of $15.0 \mathrm{~mm} /$ day. The other isolates displayed higher growth rates, varying between 16.5 and $17.7 \mathrm{~mm} /$ day (Fig. 3A).

Molecular characterization and phylogenetic analysis of the Pestalotiopsis isolates. The ITS sequences of the group I, II, and III isolates were amplified by PCR and the target bands with lengths of 506 to $586 \mathrm{bp}$ were purified, sequenced, and submitted to the NCBI database (Table 1). BLASTn searches showed that they shared the highest similarities with those of $P$. theae (group I isolates), $P$. camelliae (group II isolates), and $P$. clavispora (group III isolates), respectively (Table 1$)$.

Sequence alignment showed that 34 ITS sequences were classified into three different groups, which was consistent with the classification of the isolates by culturing features (Supplementary Fig. S1). Nucleotide differences were primarily observed in the positions of
A

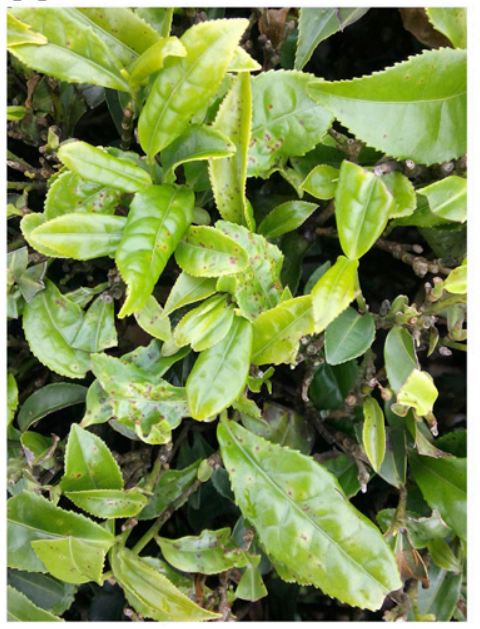

B

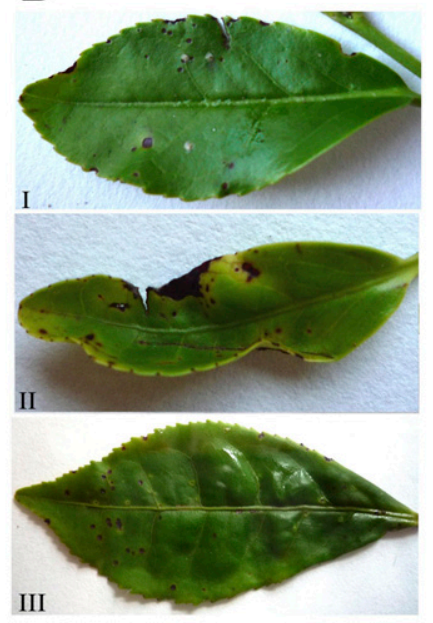

C
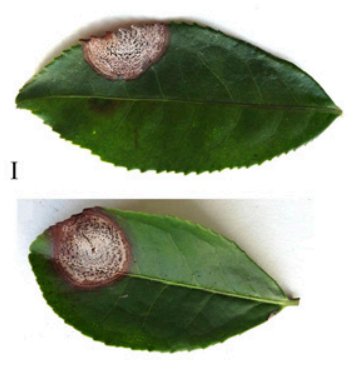

II

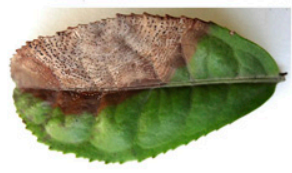

Fig. 1. Typical symptoms of brown-black spot and gray blight diseases on leaves of Camellia sinensis (L.) O. Kuntze. A, tender leaves seriously infected by brown-black spot disease in the field in Xuan'en county; B, typical symptoms of brown-black spot disease on the leaves collected from Xuan'en, Yichang, and Yingshan counties, Hubei Province, P. R. China, respectively; C, typical symptoms of gray blight disease on the tea leaves collected from Yingshan, Yichang, and Zhuxi counties, Hubei Province, respectively.

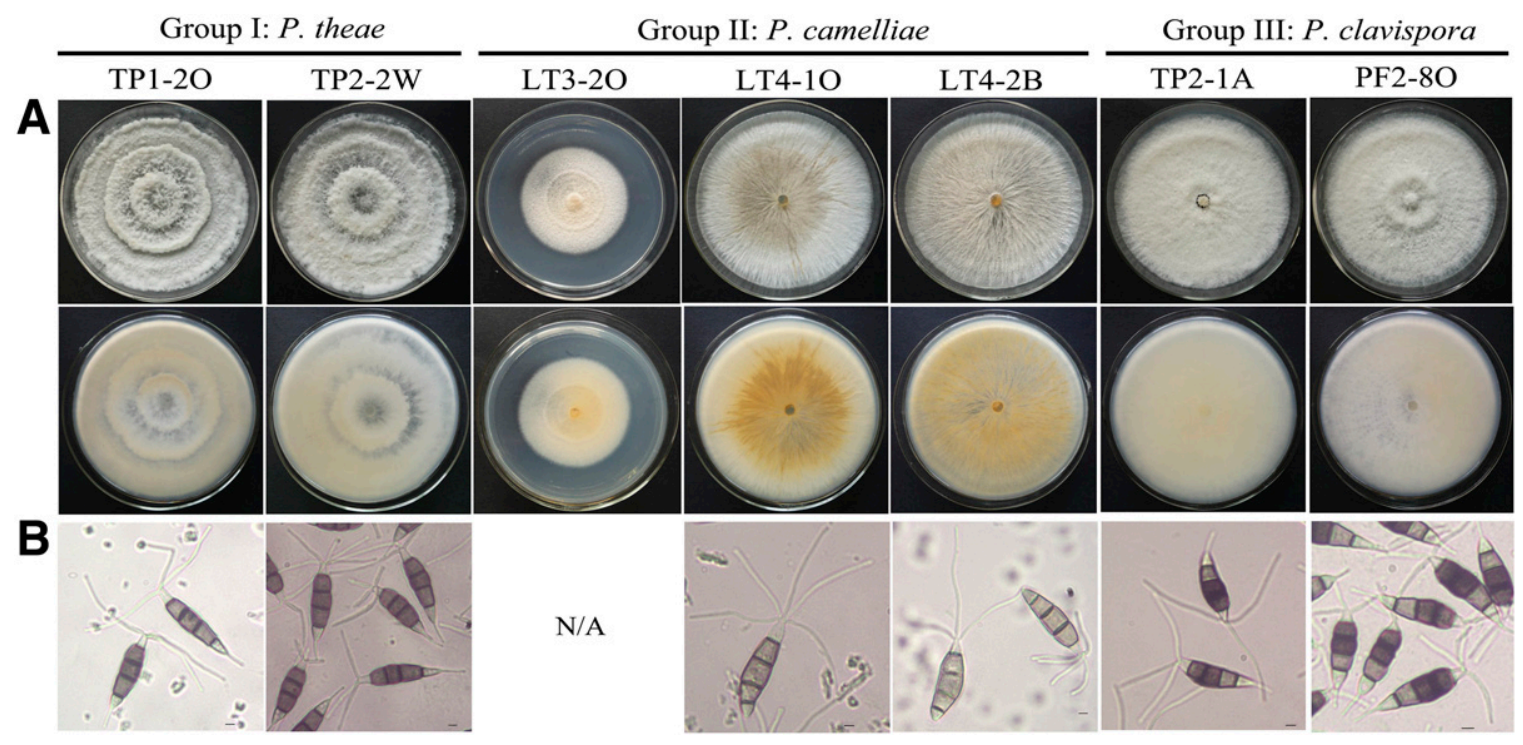

Fig. 2. Morphologies of the representative isolates belonging to Pestalotiopsis spp. obtained in this study. $\mathbf{A}$, views of the surface and the bottom of each PDA plate of representative isolates. B, conidia (scale bar $=10 \mu \mathrm{m}$ ) were produced by these isolates on PDA at a prolonged culture time (over 10 days). "N/A" means no production of conidium under the tested conditions. 
Table 1. Information of the Pestalotiopsis isolates obtained in this study and their BLASTn results

\begin{tabular}{|c|c|c|c|c|c|c|c|c|c|c|}
\hline Isolate $^{a}$ & Species & Region $^{b}$ & Sequence $^{c}$ & $\begin{array}{c}\text { Accession } \\
\text { no. }^{d}\end{array}$ & $\begin{array}{l}\text { Total } \\
\text { score }\end{array}$ & $\begin{array}{c}\text { Coverage } \\
(\%)\end{array}$ & E value & $\begin{array}{c}\text { Identity } \\
(\%)\end{array}$ & $\begin{array}{c}\text { Accession } \\
\text { no. }^{e}\end{array}$ & Isolate $^{f}$ \\
\hline TP1-10 & P. theae & Yichang & ITS & MF115525 & 913 & 100 & 0 & 99 & KM513607 & JS-2 \\
\hline TP1-2O & P. theae & Yichang & ITS & MF115526 & 907 & 100 & 0 & 99 & KM513607 & JS-2 \\
\hline TP1-1C & P. theae & Yichang & ITS & MF115527 & 920 & 100 & 0 & 100 & KM513607 & JS-2 \\
\hline TP1-2G & P. theae & Yichang & ITS & MF115528 & 920 & 100 & 0 & 100 & KM513607 & JS-2 \\
\hline TP1-2-E & P. theae & Yichang & ITS & MF115529 & 920 & 100 & 0 & 100 & KM513607 & JS-2 \\
\hline TP2-1W & P. theae & Yichang & ITS & MF115530 & 920 & 100 & 0 & 100 & KM513607 & JS-2 \\
\hline TP2-2W & P. theae & Yichang & ITS & MF115531 & 920 & 100 & 0 & 100 & KM513607 & JS-2 \\
\hline TP2-2W & P. theae & Yichang & $T U B$ & MF115571 & 773 & 93 & 0 & 99 & DQ137868 & PSH2000I-099 \\
\hline TP2-2W & P. theae & Yichang & $T E F$ & MF115580 & 435 & 100 & $4 \mathrm{e}^{-118}$ & 93 & AB453847 & TAP360105 \\
\hline YS4-1 & P. theae & Yingshan & ITS & MF115532 & 920 & 100 & 0 & 100 & KM513607 & JS-2 \\
\hline YS5-1 & P. theae & Yingshan & ITS & MF115533 & 915 & 100 & 0 & 99 & KM513607 & JS-2 \\
\hline ZX4-2 & P. theae & Zhuxi & ITS & MF115534 & 915 & 100 & 0 & 99 & KM513607 & JS-2 \\
\hline TP3-2A & P. theae & Yichang & ITS & MF115535 & 915 & 100 & 0 & 99 & KM513607 & JS-2 \\
\hline TP3-2A & P. theae & Yichang & $T U B$ & MF115572 & 778 & 93 & 0 & 100 & DQ137868 & PSH2000I-099 \\
\hline TP3-2A & P. theae & Yichang & $T E F$ & MF115581 & 440 & 100 & $9 \mathrm{e}^{-120}$ & 93 & AB453847 & TAP360105 \\
\hline WS3-2 & P. theae & Yingshan & ITS & MF115536 & 902 & 99 & 0 & 99 & KM513607 & JS-2 \\
\hline WS3-12 & P. theae & Yingshan & ITS & MF115537 & 902 & 99 & 0 & 99 & KM513607 & JS-2 \\
\hline TP1-1E & P. theae & Yichang & ITS & MF115538 & 913 & 100 & 0 & 99 & KM513607 & JS-2 \\
\hline YS1-1 & P. theae & Yingshan & ITS & MF115539 & 913 & 100 & 0 & 99 & KM513607 & JS-2 \\
\hline YS2-1 & P. theae & Yingshan & ITS & MF115540 & 913 & 100 & 0 & 99 & KM513607 & JS-2 \\
\hline PF3-1 & P. theae & Xuan'en & ITS & MF115541 & 909 & 100 & 0 & 99 & JN943624 & 3.9188 \\
\hline YC5-1 & P. theae & Yichang & ITS & MF115542 & 922 & 100 & 0 & 100 & HQ832793 & LH13 \\
\hline YC5-1 & P. theae & Yichang & $T U B$ & MF115573 & 774 & 94 & 0 & 99 & DQ137872 & PSHI2002Endo312 \\
\hline YC5-1 & P. theae & Yichang & $T E F$ & MF115582 & 435 & 100 & $4 \mathrm{e}^{-118}$ & 93 & AB453847 & TAP360105 \\
\hline YC6-3 & P. theae & Yichang & ITS & MF115543 & 922 & 100 & 0 & 100 & HQ832793 & LH13 \\
\hline YC6-3 & P. theae & Yichang & $T U B$ & MF115574 & 778 & 93 & 0 & 100 & DQ137872 & PSHI2002Endo312 \\
\hline YC6-3 & P. theae & Yichang & $T E F$ & MF115583 & 435 & 100 & $4 \mathrm{e}^{-118}$ & 93 & AB453847 & TAP36O105 \\
\hline ZX1-1 & P. theae & Zhuxi & ITS & MF115544 & 922 & 100 & 0 & 100 & HQ832793 & LH13 \\
\hline ZX5-2 & P. theae & Zhuxi & ITS & MF115545 & 922 & 100 & 0 & 100 & JN943624 & 3.9188 \\
\hline ZX1-2 & P. theae & Zhuxi & ITS & MF115546 & 917 & 100 & 0 & 99 & HQ832793 & LH13 \\
\hline YC2-1 & P. theae & Yichang & ITS & MF115547 & 922 & 99 & 0 & 100 & JN943624 & 3.9188 \\
\hline YC2-1 & P. theae & Yichang & $T U B$ & MF115575 & 749 & 96 & 0 & 98 & DQ137868 & PSH2000I-099 \\
\hline YC2-1 & P. theae & Yichang & $T E F$ & MF115584 & 435 & 100 & $4 \mathrm{e}^{-118}$ & 93 & AB453847 & TAP360105 \\
\hline YC1-1 & P. theae & Yichang & ITS & MF115548 & 905 & 100 & 0 & 99 & HQ832793 & LH13 \\
\hline WS1-2 & P. theae & Yingshan & ITS & MF115549 & 863 & 98 & 0 & 98 & KM513607 & JS-2 \\
\hline WS1-10 & P. theae & Yingshan & ITS & MF115550 & 863 & 98 & 0 & 98 & KM513607 & JS-2 \\
\hline JF12-3 & P. camelliae & Xuan'en & ITS & MF115551 & 822 & 100 & 0 & 94 & KY244076 & INTA-IMC1359 \\
\hline LT3-2O & P. camelliae & Xuan'en & ITS & MF115552 & 1000 & 99 & 0 & 99 & KM199336 & CBS 443.62 \\
\hline LT3-2A & P. camelliae & Xuan'en & ITS & MF115553 & 1000 & 99 & 0 & 99 & KM199336 & CBS 443.62 \\
\hline LT4-1G & P. camelliae & Xuan'en & ITS & MF115554 & 1009 & 100 & 0 & 100 & KM199336 & CBS 443.62 \\
\hline LT4-3B & P. camelliae & Xuan'en & ITS & MF115555 & 1003 & 100 & 0 & 99 & KM199336 & CBS 443.62 \\
\hline LT4-3B & P. camelliae & Xuan'en & $T U B$ & MF115576 & 754 & 94 & 0 & 99 & KX895303 & LC3348 \\
\hline LT4-3B & P. camelliae & Xuan'en & $T E F$ & MF115585 & 527 & 100 & $7 e^{-146}$ & 99 & KX895176 & LC3154 \\
\hline LT4-2B & P. camelliae & Xuan'en & ITS & MF115556 & 1003 & 99 & 0 & 100 & KM199336 & CBS 443.62 \\
\hline LT4-2C & P. camelliae & Xuan'en & ITS & MF115557 & 1003 & 99 & 0 & 100 & KM199336 & CBS 443.62 \\
\hline LT4-1 & P. camelliae & Xuan'en & ITS & MF115558 & 1000 & 99 & 0 & 99 & KM199336 & CBS 443.62 \\
\hline LT4-2 & P. camelliae & Xuan'en & ITS & MF115559 & 937 & 100 & 0 & 98 & KM199336 & CBS 443.62 \\
\hline LT4-2A & P. camelliae & Xuan'en & ITS & MF115560 & 937 & 100 & 0 & 98 & KM199336 & CBS 443.62 \\
\hline LT4-1O & P. camelliae & Xuan'en & ITS & MF115561 & 769 & 98 & 0 & 92 & KX894934 & LC3003 \\
\hline LT4-1O & P. camelliae & Xuan'en & $T U B$ & MF115577 & 617 & 94 & $6 e^{-173}$ & 93 & KX895303 & LC3348 \\
\hline LT4-1O & P. camelliae & Xuan'en & $T E F$ & MF115586 & 538 & 100 & $3 \mathrm{e}^{-149}$ & 99 & KX895176 & LC3154 \\
\hline LT4-1B & P. camelliae & Xuan'en & ITS & MF115562 & 769 & 98 & 0 & 92 & KX894934 & LC3003 \\
\hline $\mathrm{TP} 2-1 \mathrm{~N}$ & P. clavispora & Yichang & ITS & MF115563 & 1778 & 98 & 0 & 100 & KX866908 & KFRD-68 \\
\hline $\mathrm{TP} 2-1 \mathrm{~N}$ & P. clavispora & Yichang & $T U B$ & MF115578 & 771 & 95 & 0 & 99 & KX721073 & MAC-01 \\
\hline $\mathrm{TP} 2-1 \mathrm{~N}$ & P. clavispora & Yichang & $T E F$ & MF115587 & 532 & 100 & $1 \mathrm{e}^{-147}$ & 99 & KM402034. & ZJXJ-PA1 \\
\hline TP2-1A & P. clavispora & Yichang & ITS & MF115564 & 1778 & 98 & 0 & 100 & KX866908 & KFRD-68 \\
\hline $\mathrm{TP} 2-4 \mathrm{C}$ & P. clavispora & Yichang & ITS & MF115565 & 909 & 100 & 0 & 100 & KX866908 & KFRD-68 \\
\hline PF2-1 & P. clavispora & Xuan'en & ITS & MF115566 & 909 & 100 & 0 & 100 & KX866908 & KFRD-68 \\
\hline PF2-1 & P. clavispora & Xuan'en & $T U B$ & MF115579 & 773 & 96 & 0 & 99 & KX721073 & MAC-01 \\
\hline PF2-1 & P. clavispora & Xuan'en & $T E F$ & MF115588 & 527 & 100 & $6 e^{-146}$ & 99 & KM402034 & ZJXJ-PA1 \\
\hline PF2-8A & P. clavispora & Xuan'en & ITS & MF115567 & 902 & 100 & 0 & 99 & KX866908 & KFRD-68 \\
\hline PF2-8O & P. clavispora & Xuan'en & ITS & MF115568 & 857 & 100 & 0 & 98 & KР689177 & FL21 \\
\hline MA1-2 & P. clavispora & Xuan'en & ITS & MF115569 & 739 & 98 & 0 & 94 & KM438014 & UMT85-K \\
\hline MA3-1 & P. clavispora & Xuan'en & ITS & MF115570 & 739 & 98 & 0 & 94 & KM438014 & UMT85-K \\
\hline
\end{tabular}

a The isolates obtained in this study.

${ }^{\mathrm{b}}$ Regions where the samples were collected in Hubei Province.

c Sequences of ITS, TUB, and TEF, respectively.

${ }^{\mathrm{d}}$ GenBank accession numbers for the isolates obtained in this study.

e GenBank accession numbers for the isolates obtained in NCBI.

$\mathrm{f}$ The isolates registered in NCBI with the best matches with those obtained in this study. 
47 to 118,372 to 396 , and 461 to 480 nt. Specifically, sequences in the group of $P$. theae had an $8 \mathrm{nt}$ deletion in the position of 377 to 385 $\mathrm{nt}$, in the group of $P$. camelliae a 34 to $36 \mathrm{nt}$ insertion in 58 to $92 \mathrm{nt}$, and in the group of $P$. clavispora a 51 to $52 \mathrm{nt}$ deletion in 47 to $118 \mathrm{nt}$. The nucleotide differences corresponded to the specific species $P$. theae, $P$. camelliae, and $P$. clavispora, respectively.

In the phylogenetic tree constructed by NJ method (Fig. 4), 34 ITS sequences formed three major clusters with related strains available in GenBank. Within each cluster, the sequences were highly divergent and as a result, most of them formed discrete branches (Fig. 4). For example, five isolates (LT4-2, LT4-2A, LT4-1O, LT4-1B, and JF12-3) were distantly separated from other $P$. camellia isolates, and three isolates (TP2-1A, TP2-4C, and PF2-1) were clearly separated from other $P$. clavispora isolates. Generally, each cluster corresponded to one species of $P$. theae, $P$. camelliae, or $P$. clavispora matching to groups I, II, and III, respectively (Figs. 2 and 4). Six representative isolates (TP2-2W, TP3-2A; TP2-1 N, PF2-1; and LT4-3B, LT4-1O) belonging to the three species were further subjected to sequencing their partial TUB and TEF sequences. BLASTn searches also indicated that they shared the highest similarities with those of $P$. theae, $P$. camelliae, and $P$. clavispora, respectively (Table 1). Phylogenetic analysis based on the concatenated sequences including ITS, TUB, and TEF sequences confirmed the identification of the species (Fig. 5B).

Pathogenicity tests of the Pestalotiopsis isolates. Pathogenicity analysis of the Pestalotiopsis isolates were initially tested on detached leaves of $C$. sinensis var. Taicha No. 12. Under the condition of needling, inoculation of agar discs without mycelia did not result in any symptoms on tea leaves; in contrast, inoculation of mycelial discs caused apparent lesions for the isolates with high virulence at 1 day post inoculation (dpi). The lesions were brown, and the lengths varied significantly among three species (Figs. 3B and 5A). At 3 dpi on wounded leaves, representative isolates of $P$. theae and $P$. clavispora almost all spread to the leaf side edges and caused larger lesions (12.3 to $16.2 \mathrm{~mm}$ in length) than the representative isolates of $P$. camellia, which resulted in much smaller lesions ( 0.7 to $3.7 \mathrm{~mm}$ in length) (Figs. $3 \mathrm{~B}$ and 5A). Noticeably, brown-black spots with diameters of 1 to $2 \mathrm{~mm}$ were also formed at the edge of all lesions (Fig. 5A and C), which was similar to the symptoms observed in the field (Figs. 1 and 5CI, II). However, for the unwounded leaves, only TP1-2O and TP2-2W of $P$. theae were able to induce lesions that were generally small $(6.7$ and $7.5 \mathrm{~mm}$ in length, respectively) and accompanied by brown-black spots with sizes of 1 to $2 \mathrm{~mm}$ (Fig. 5A). Like the control, five isolates belonging to $P$. camellia and $P$. clavispora could not cause any disease symptoms on unwounded leaves (Fig. 5A).
The conidium suspension of TP1-2O and TP2-2W ( $P$. theae) did not induce any disease symptoms on unwounded leaves, while after wounding they caused brown lesions (Fig. 5B) of 4.4 and $12.4 \mathrm{~mm}$, respectively. Such lesions were surrounded by brown-black spots (Fig. 5CIII), which was similar to the symptoms of natural infection (Figs. 1A and B) or those obtained with mycelial discs (Figs. 5CI and II).

Pathogenicity analysis of TP2-2W of $P$. theae, PF2-8 of $P$. clavispora, and LT4-2B of $P$. camellia were further tested on intact leaves with a conidium suspension in the field. At 20 dpi with TP2-2W of $P$. theae, small brown-black spots clearly appeared on the leaves and remained small until the experiments ended at $30 \mathrm{dpi}$ (Fig. 5E), matching well with the original ones that had been observed. However, no obvious symptoms were observed for the PF2-8 of P. clavispora, LT4-2B of $P$. camellia, and the control (data not shown).

The reobtained isolates from the inoculation-induced diseased leaves matched well with the ones for inoculation, as indicated by the colony morphology, microscopic observation, and their identical ITS sequences.

Characterization of isolates related to tea gray blight disease. To compare the molecular and biological features of the isolates responsible for the brown-black spot disease (designated as BSD isolates) and those for the gray blight disease (designated as GBD isolates), 12 leaf samples showing gray blight disease symptoms (Fig. 1C) were collected from the same (Yichang and Yingshan counties) and different (Zhuxi county) regions at almost the same time (within the same week) with the samples showing the brown-black spot disease symptoms, and were subjected to pathogen isolation by culturing the conidia picked from acervuli over the lesions. As expected, $P$. theae colonies, which were identified by the morphologies and ITS sequences (see below), were abundantly isolated. A P. theae isolate was taken from each leaf sample, and three to four isolates were selected for the samples from each county (12 in total, designated as YC-, YS-, and ZX-isolates according to their origins; Table 1) for further characterization. After culturing on PDA in the dark at $25^{\circ} \mathrm{C}$, all the GBD isolates showed the macro-morphology, conidium conformation, and color similar to those of BSD isolates (Fig. 2A and B). However, the conidia of the GBD isolates were obviously longer than those of the BSD isolates (30.6 to 32.3 versus 24.3 to $24.6 \mu \mathrm{m}$ in average length) (Table 2). The GBD isolates showed significantly varying growth rates ranging from 9.2 to $18.0 \mathrm{~mm} /$ day, which covered the growth rate range of the BSD isolates (Supplementary Fig. S2). On the ITS phylogenetic tree, YC- and ZX-isolates (except for ZX4-2) were clearly separated from BSD isolates, and were closely related to the tea endophytic isolates (PSHI20002Endo310 and PSHI20002Endo312) collected from Yunnan

Table 2. Morphological characteristics of the representative isolates of Pestalotiopsis spp. obtained in this study and the referenced ones

\begin{tabular}{|c|c|c|c|c|c|c|c|c|}
\hline \multirow{2}{*}{$\begin{array}{l}\text { Species and } \\
\text { group }\end{array}$} & \multirow[b]{2}{*}{ Isolate } & \multicolumn{2}{|c|}{$\begin{array}{l}\text { Conidium size } \\
\text { ranges }^{\mathbf{a}}(\mu \mathbf{m})\end{array}$} & \multirow{2}{*}{$\begin{array}{c}\text { Number } \\
\text { of apical } \\
\text { appendages }\end{array}$} & \multirow{2}{*}{$\begin{array}{c}\text { Length of apical } \\
\text { appendages } \\
(\mu \mathrm{m})\end{array}$} & \multirow{2}{*}{$\begin{array}{l}\text { Morphology } \\
\text { of apical } \\
\text { appendages }\end{array}$} & \multirow{2}{*}{$\begin{array}{c}\text { Color of } \\
\text { median cells }\end{array}$} & \multirow{2}{*}{$\begin{array}{l}\text { Length of basal } \\
\text { appendages } \\
(\mu \mathrm{m})\end{array}$} \\
\hline & & Length & Width & & & & & \\
\hline \multirow[t]{2}{*}{ P. theae (group I) } & TP1-2O & $20.1 \sim 24.6 \sim 27.8$ & $3.7 \sim 5.6 \sim 7.7$ & $2 \sim 3$ & $19.2 \sim 29.7 \sim 39.8$ & \multirow[t]{7}{*}{ Knobbed } & \multirow{7}{*}{$\begin{array}{r}\text { Concolorous, } \\
\text { dark brown }\end{array}$} & $3.6 \sim 7.1 \sim 11.3$ \\
\hline & TP2-2W & $20.5 \sim 24.3 \sim 30.9$ & $5.2 \sim 6.9 \sim 8.3$ & $2 \sim 4$ & $15.8 \sim 29.8 \sim 41.4$ & & & $5.0 \sim 6.9 \sim 8.7$ \\
\hline \multirow[t]{3}{*}{ P. theae $\left(\mathrm{GLD}^{\mathrm{d}}\right)$} & YS-2-1 & $22.8 \sim 30.6 \sim 35.6$ & $3.5 \sim 6.2 \sim 8.2$ & $2 \sim 3$ & $14.8 \sim 28.2 \sim 37.6$ & & & $2.1 \sim 6.5 \sim 9.1$ \\
\hline & YC-6-3 & $21.9 \sim 31.8 \sim 37.0$ & $4.9 \sim 7.4 \sim 10.3$ & $2 \sim 4$ & $4.4 \sim 21.7 \sim 50.2$ & & & $3.7 \sim 7.5 \sim 10.9$ \\
\hline & ZX-5-1 & $26.0 \sim 32.3 \sim 42.7$ & $4.5 \sim 6.6 \sim 8.3$ & $2 \sim 4$ & $16.3 \sim 31.2 \sim 45.6$ & & & 4.5 7.9 9.9 \\
\hline \multirow[t]{2}{*}{ P. theae $\left(\mathrm{ref}^{\mathrm{e}}\right)$} & & $24.8-29.5$ & $6.6-8.3$ & $2-3$ & $23.6-42.5$ & & & $5.9-9.4$ \\
\hline & & $23-35$ & $5.5-8$ & $2-4$ & $25-60$ & & & \\
\hline \multirow[t]{2}{*}{ P. camelliae (group II) } & LT4-1O & $28.8 \sim 33.0 \sim 38.0$ & 5.6 7.1 8.4 & $2 \sim 5$ & 15.5 24.9 44.8 & \multirow[t]{3}{*}{ Tubular } & \multirow{3}{*}{$\begin{array}{r}\text { Concolorous, } \\
\text { light brown }\end{array}$} & 0 \\
\hline & LT4-2B & $24.2 \sim 30.0 \sim 34.3$ & $6.4 \sim 7.8 \sim 8.8$ & $2 \sim 5$ & $16.0 \sim 27.5 \sim 38.7$ & & & 0 \\
\hline P. camelliae $\left(\right.$ ref $\left.^{\mathrm{f}}\right)$ & & $27-28.5-33$ & $7.5-8.5-9.3$ & $3-6$ & $13.5-23.8-34$ & & & 0 \\
\hline \multirow[t]{2}{*}{ P. clavispora (group III) } & TP2-1A & $19.8 \sim 22.8 \sim 26.4$ & $4.9 \sim 6.6 \sim 7.8$ & $2 \sim 3$ & $15.2 \sim 22.2 \sim 34.1$ & \multirow[t]{3}{*}{ Tubular } & \multirow{3}{*}{$\begin{array}{l}\text { Two dark } \\
\text { brown, one } \\
\text { light brown }\end{array}$} & $4.1 \sim 6.3 \sim 8.3$ \\
\hline & PF2-8O & $19.0 \sim 23.1 \sim 26.312$ & $5.4 \sim 6.9 \sim 8.7$ & $2 \sim 4$ & $14.4 \sim 22.8 \sim 29.3$ & & & $4.7 \sim 7.0 \sim 9.3$ \\
\hline P. clavispora $\left(\right.$ ref $\left.^{\mathrm{f}}\right)$ & & $21.2-27.1$ & $5.2-7.1$ & $2-3$ & $16.5-26.5$ & & & $1.8-4.2$ \\
\hline
\end{tabular}

${ }^{a}$ Minimum, average, and maximum length and width of 50 conidia recorded from each selected Pestalotiopsis spp. from China.

${ }^{\mathrm{b}}$ Minimum, average, and maximum length of apical appendages of 50 conidia.

${ }^{\mathrm{c}}$ Minimum, average, and maximum length of basal appendages of 50 conidia.

${ }^{\mathrm{d}}$ Isolates related to tea gray blight disease.

e The referenced species according to Ge et al. 2009.

f The referenced species according to Zhang et al. 2012. 
Province and GBD isolates (PSH20001-083 and PSH20001-099) collected from Guangxi and Zhejiang provinces, respectively (Ge et al. 2009). However, YS-isolates were clustered together with BSD isolates (Fig. 4; Table 1). Unlike BSD isolates, GBD isolates showed no virulence on detached tender leaves of $C$. sinensis var. Taicha No. 12 under unwounded conditions, whereas they induced lesions with lengths ranging from 3.5 to $29.8 \mathrm{~mm}$ after wounding. Unlike BSD isolates, GBD isolates did not result in the appearance of brown-black spots along with the induction of brown lesions on tender tea leaves (Fig. 5D).

\section{Discussion}

In this study, brown-black spot disease was described as a novel tea leaf disease as it causes apparent symptoms on tender tea leaves that are quite different from those previously reported (Z. M. Chen and X. L. Sun 2013; Maharachchikumbura et al. 2014). Typical diseased samples were collected from three main tea cultivation regions of Hubei, China, and the related pathogens were isolated and characterized. The results showed that Pestalotiopsis fungi (34 isolates, see the typical conidia of a Pestalotiopsis species in Fig. 2B: fusiform, 5-celled, brown median cells, and hyaline end cells, appendages) were dominant among all fungi isolated from the diseased samples, though Phomopsis (12 isolates) and Colletotrichum fungi (eight isolates) were also isolated simultaneously. The colony and microscopic characteristics suggested that the Pestalotiopsis isolates might belong to $P$. theae, $P$. camelliae, and $P$. clavispora, respectively, according to the standards described by Ge et al. (2009) and Guba (1961). The results of phylogenetic analysis confirmed those of morphological characterization. Hence, the isolates in groups I, II, and III were identified to belong to $P$. theae, $P$. camelliae, and P. clavispora, respectively (Fig. 2). No matter what mycelial discs or conidium suspensions were used, inoculation of the Pestalotiopsis fungi could result in brown-black spots (1 to $2 \mathrm{~mm}$ in diameter), which were similar to the symptoms observed in the field. In contrast, inoculation of the Phomopsis and Colletotrichum fungi could not cause any disease symptoms, though they were found to be the causal agents of tea leaf brown blight disease in China (Y. J. Chen et al. 2016; Guo et al. 2014). The results obtained through the identification of these pathogens in microscopy and using various molecular tools, as well all the assays conducted to fulfill Koch's postulates, clearly show that $P$. theae,

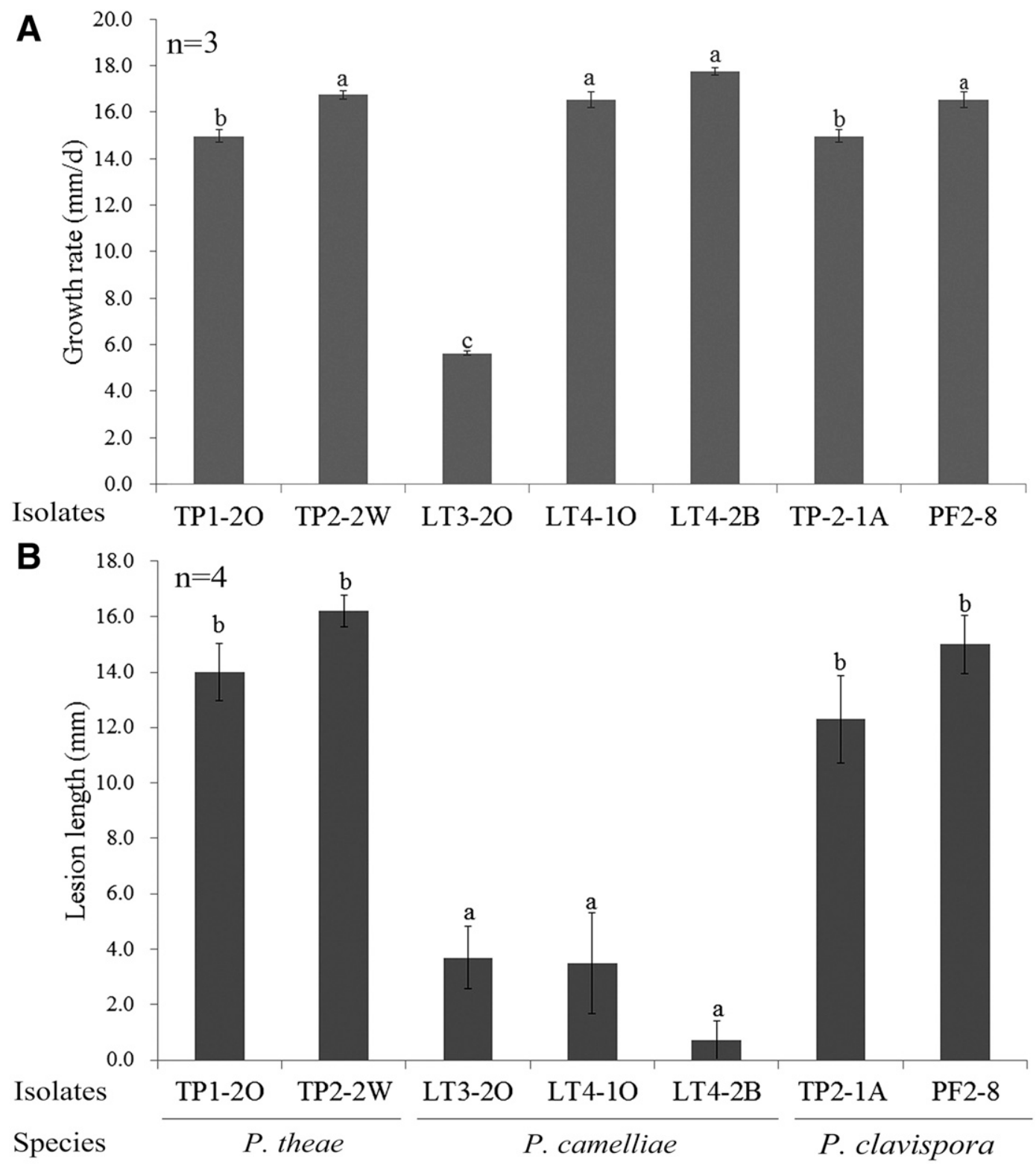

Fig. 3. Growth rates and virulence of the representative isolates of $P$. theae, $P$. camelliae, and $P$. clavispora. A, growth rates were accessed by culturing a mycelium plugs on PDA plates in the dark at $25^{\circ} \mathrm{C}$ at 4 days post inoculation (dpi). B, lesion lengths on wounded tender leaves of var. Taicha No. 2 induced by inoculation of their mycelial discs at $25^{\circ} \mathrm{C}$ for a light period of $12 \mathrm{~h}$ at $3 \mathrm{dpi}$. Data were analyzed with SPSS Statistics 21.0 (WinWrap Basic; http://www.winwrap.com) by one-way ANOVA, and means were compared using Tukey's test at a significance level of $P=0.05$. Letters over the bars indicate the significant difference at $P=0.05$. Replicates for the experiments are indicated in the panels. 

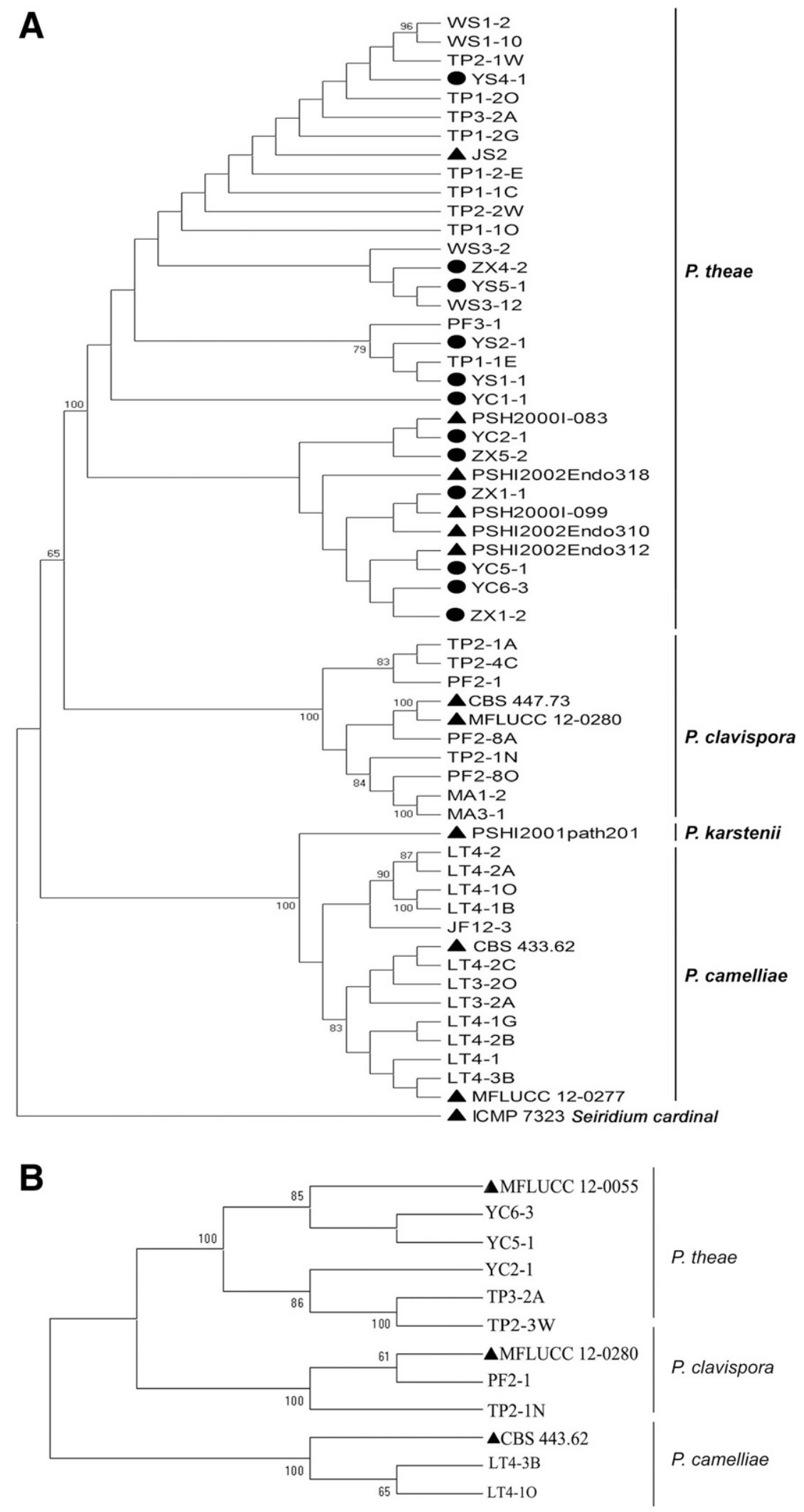

Fig. 4. Phylogenetic analysis of ITS sequences and concatenated ITS, TUB, and TEF sequences of the Pestalotiopsis isolates obtained in this study and those chosen from GenBank. The evolutionary history was conducted in MEGA 5.2.2 and inferred using the neighbor-joining method. A and B, phylogenetic analysis based on ITS sequences and concatenated ITS, TUB, and TEF sequences, respectively. The referenced species of Pestalotiopsis were chosen according to the BLASTn results and indicated by triangles $(\boldsymbol{\Lambda})$. An isolate of Seiridium cardinal was involved as outgroup. Bootstrap support values (more than 60\%) are shown at the nodes. The GenBank accession numbers for the ITS sequences of the isolates obtained in this study are listed in Table 1, and of the reference isolates: JS2 (KM513607), PSH2000I-083 (AY681477), PSH2000I-099 (AY681478), PSHI2002Endo312 (AY681481), PSHI2002Endo310 (AY681480), PSHI2002Endo318 (AY681482), MFLUCC 12-0280 (JX398978), CBS 447.73 (KM199374), PSHI2001path201 (AY681472), CBS 433.62 (KM199336), MFLUCC 12-0277 (NR_120188), ICMP 7323 (AF409995); and for the ITS, TUB, and TEF sequences: MFLUCC 12-0055 (JQ683727, JQ683711, JQ683743), MFLUCC 12-0280 (JX398978, JX399013, JX399044), CBS 443.62 (KM199336, KM199424, KM199512). The evolutionary history was conducted in MEGA 5.2.2. Bootstrap support values (more than 60\%) are shown at the nodes. The isolates were collected from the samples showing the tea gray blight disease symptoms (GBD isolates) from Yichang (YC-) and Zhuxi (ZX-) counties, Hubei Province, P. R. China, respectively, and indicated by circles $(\mathbf{O})$. The remaining isolates that are not indicated were obtained from the leaf samples showing brown-black spot symptoms. 
$P$. camellia, and $P$. clavispora were the dominant fungi associated with brown-black spots on tea tender leaves. Although P. camellia and P. clavispora showed no virulence on tea leaves on unwounded leaves, we concluded that they were still closely associated with the disease, considering their high incidence in the diseased plants. Both species might induce severe disease by invading tea plants via wounds with assistance from insects, mechanical damage, or other unknown factors, which had been exemplified by Phomopsis species (Bai et al. 2015). The results also confirmed that this disease is quite different from the two tea diseases reported previously, namely white scab disease and brown round spot disease. The latter two always cause lesions with a grayish appearance in the center on tender tea leaves, while this gray part is always absent on leaves affected by small brown-black spot. It has also been shown that white scab is caused by Phyllosticta theaefolia of Sphaeriales, Ascomycota, and the brown round spot disease by Cercospora theae of Moniliales, Deuteromycotina (Z. M. Chen and X. L. Sun 2013).
Morphological characteristics, such as conidium sizes and apical appendage length of Pestalotiopsis, may vary responsively with the changes of culture conditions. In many cases, simple observation of morphology is not sufficient to distinguish the members of genus Pestalotiopsis (Jeewon et al. 2003). Molecular characterization of fungal genes, such as ITS, TUB, and TEF, can reveal more specific differences between the Pestalotiopsis fungi (Maharachchikumbura et al. 2014). In this study, 34 isolates were firstly subjected to phylogenetic analysis of ITS sequences using NJ method. The results revealed that these isolates clustered together with the species of $P$. theae, P. camelliae, and P. clavispora, respectively (Fig. 4A), which was also confirmed by the results of ML method (Supplementary Fig. S3). In both phylogenetic trees, some isolates (LT4-2, LT4-2A, LT41O, LT4-1B, JF12-3) were located between species $P$. camelliae and $P$. karstenii (possibly a synonym of $P$. maculans, Jeewon et al. 2003), but they could be easily distinguished from $P$. karstenii according to
A
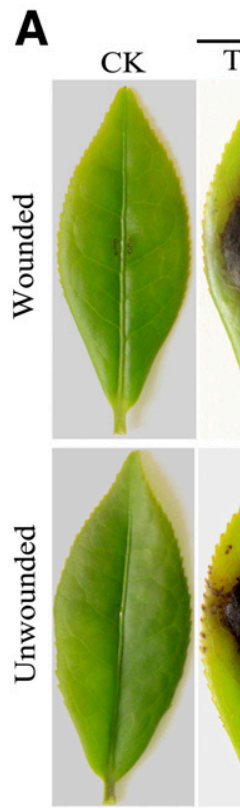

C

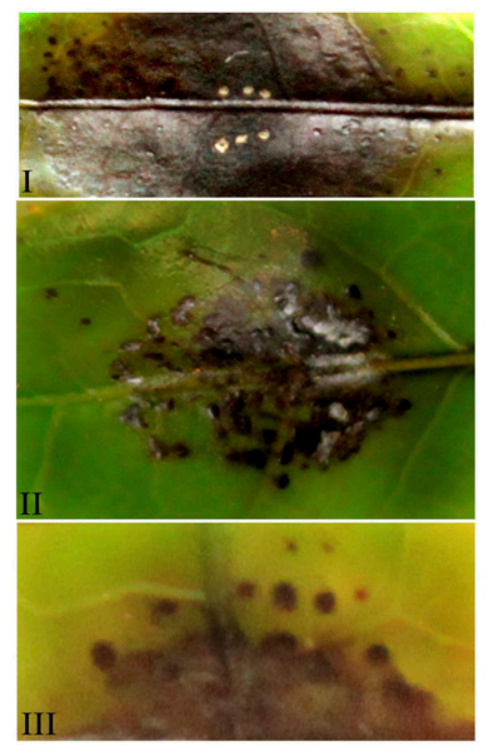

P. theae

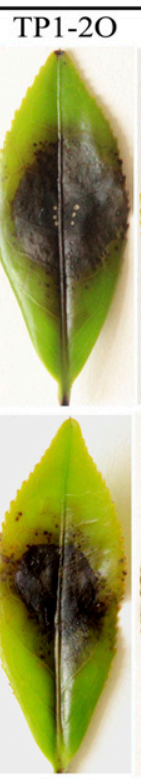

TP2-2W

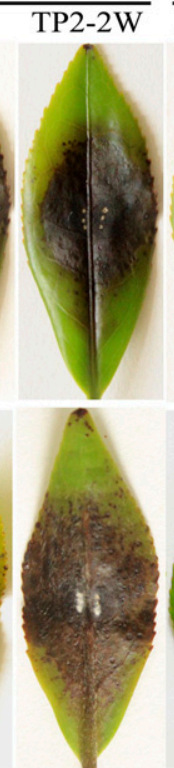

is

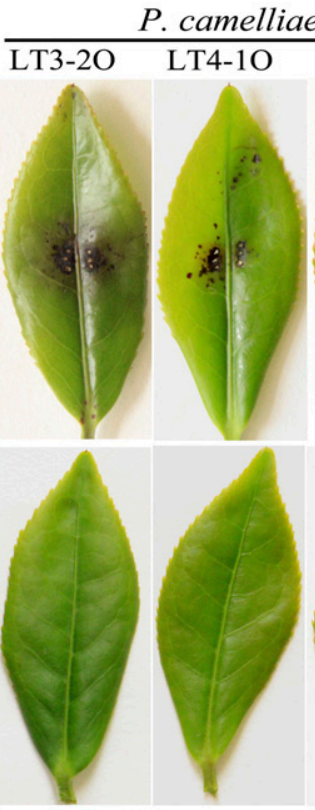

D

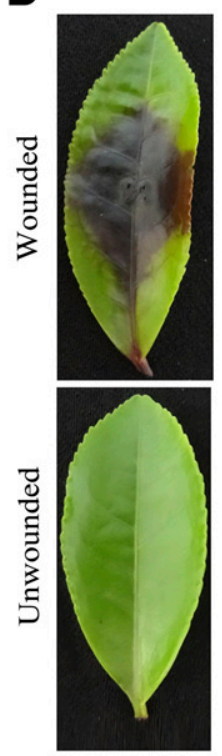

P. clavispora

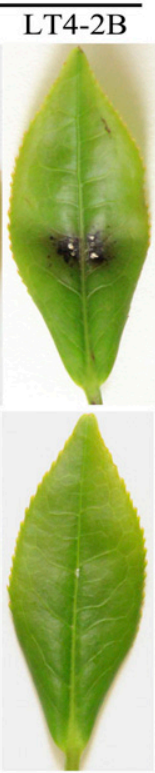

$\mathbf{E}$

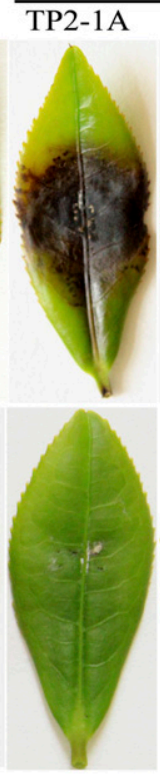

TP2-2W
B
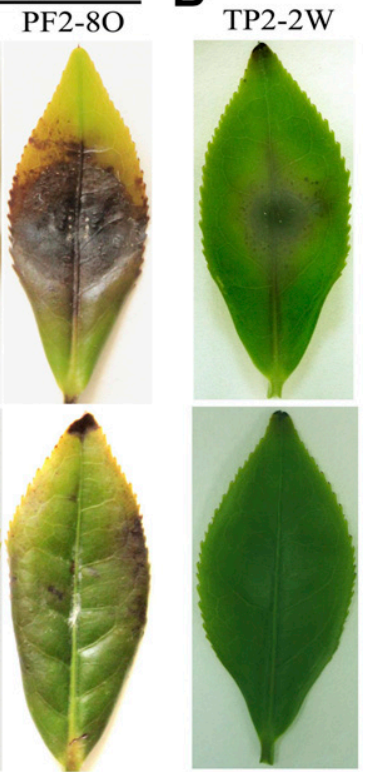

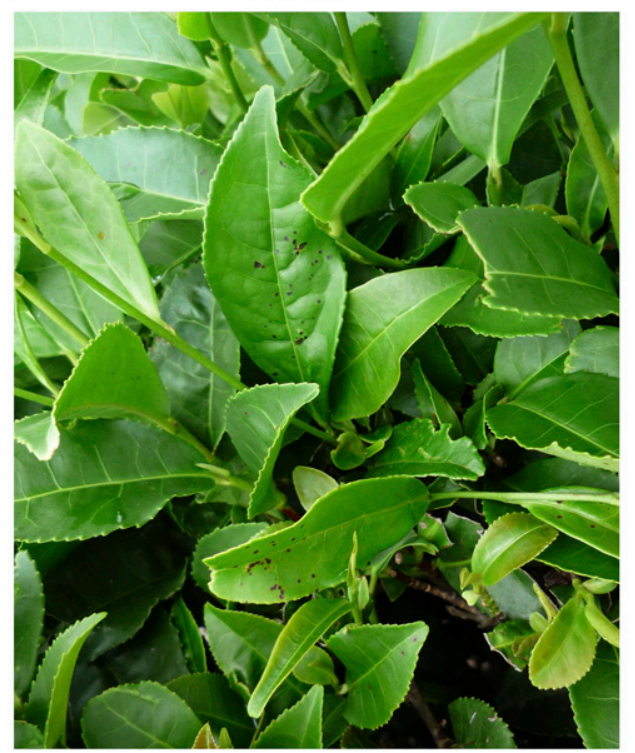

Fig. 5. Typical symptoms on tender leaves of var. Taicha No. 2 induced by inoculation of the representative isolates of $P$. theae, $P$. camelliae, and $P$. clavispora. A, symptoms induced by inoculations of mycelial discs of three Pestalotiopsis species on wounded (upper) and unwounded (below) leaves, respectively. B, symptoms induced by conidia of isolate TP22W under the both conditions, respectively. C, close view of the symptoms induced by mycelial discs of isolate TP2-2W on wounded (I) and unwounded (II) leaves, and by conidia sprayed on wounded leaves (III). D, symptoms induced by mycelial discs of ZX5-1 isolate on wounded and unwounded leaves, respectively, at 3 days post inoculation, as representative symptoms for GBD isolates. E, symptoms on intact tea leaves (C. sinensis var. Enshitaizicha) inoculated with conidium suspension (10\%/ml) of TP2-2W of $P$. theae. 
their morphological properties, such as conidium size, apical appendage number, and basal appendage-forming ability (Z. M. Chen and X. L. Sun 2013; Maharachchikumbura et al. 2014) (Table 2). The taxonomic status was further confirmed by phylogenetic analysis with $T U B$ and TEF sequences for the isolates located far from the major branch of each species (Fig. 4B). Consistent with the results of the phylogenetic analysis, the virulence of the tested isolates was also clearly delimited among different species. For example, three isolates of $P$. camelliae (LT3-2O, LT4-1O, and LT4-2B) caused similarly long but much shorter lesions than those induced by the isolates belonging to the other two species. All these results clearly demonstrate that the obtained isolates belong to $P$. theae, $P$. camelliae, and $P$. clavispora, respectively. However, these isolates showed considerably high divergence from each other within each species as shown by the NJ or ML phylogenetic tree analysis of ITS sequences (Fig. 4), suggesting that they might have complicated origins in the tea cultivation regions of Hubei.

In previous reports, the three Pestalotiopsis species had not been found to be capable of causing apparent symptoms on tender tea leaves, but $P$. theae was well characterized as a causal agent of tea gray blight disease, which attacks mature and old leaves and leads to large brown spots with the formation of apparent concentric rings in the late period of disease (see Fig. $1 \mathrm{C}$ and Z. M. Chen and X. L. Sun 2013). Here, we revealed that $P$. theae can also induce visible symptoms on tender tea leaves. It was reasonable to suspect that brown-black spot disease might be the early symptoms of gray blight disease. For this reason, we characterized the $P$. theae isolates responsible for tea gray blight disease (GBD isolates) that occurred simultaneously, and revealed that GBD isolates were distinctly different from BSD isolates in conidium sizes and pathogenicity on unwounded leaves. The BSD isolates of $P$. theae in this study are also quite different in morphology from the GBD isolates characterized in previous studies, as the conidium sizes and the lengths of apical appendages for the former exceed the bottom line of those of the latter (conidium sizes: 23 to $35 \times 5.5$ to $8 \mu \mathrm{m}$; apical appendage lengths: 25 to $60 \mu \mathrm{m})($ Z. M. Chen and X. L. Sun 2013). Additionally, BSD isolates were located in the clusters distinct from the known $P$. theae isolates previously identified from China (including tea endophytic $P$. theae isolates and previously identified BSD isolates) and most of the newly identified GBD isolates (including YC- and ZXisolates) in this study. Moreover, during a several-month observation, we found that on tender leaves of Fuyun No. 6, the small brown-lack spots caused by brown-black spot disease could not grow to large lesions as always observed with gray blight disease. More importantly, GBD isolates induced no brown-black spots on the inoculated leaves. It can be concluded that BSD isolates are possibly novel strains different from GBD isolates according to the morphologies, pathogenicity, and phylogenetic analysis, and the brown-black spot disease caused by the three Pestalotiopsis fungi is not the early stage of the gray blight disease; instead, it is a novel tea leaf disease considering the differences in symptoms and pathogens.

Pestalotiopsis fungi are commonly asexual and are extensively distributed in nature. They are classified into pathogenic, saprophytic, and endogenous types according to their pathogenicity and living habits (Ge et al. 2009). The pathogenic variants of Pestalotiopsis are considered to be economically important plant pathogens. They have a broad range of hosts and can cause plant diseases all over the world, especially in tropic and subtropic areas (Z. M. Chen and X. L. Sun 2013; Maharachchikumbura et al. 2014). P. theae can infect tea, loquat (Eriobotrya japonica), and sweet persimmon (Diospyros kaki), causing symptoms of greenish brown lesions and large grayish brown circular ringspots on leaves, respectively (Y. Chen et al. 2013; Tuset et al. 1999). This fungus was also reported to be capable of infecting camellia ( $C$. japonica), while the pathological mechanism still remains unknown (Ge et al. 2009). Unlike $P$. theae, $P$. camelliae has been little known and seems to be a new species associated with gray blight of camellia in China (Zhang et al. 2012). P. clavispora has a broader range of hosts than $P$. theae and $P$. camelliae, and causes leaf diseases on various plants, but is not pathogenic to tea leaf and is an endophyte in tea plants (Ge et al. 2009). To our knowledge, this is the first report that $P$. camelliae and $P$. clavispora can cause apparent symptoms on tender leaves.

In summary, a novel disease designated as brown-black disease, which occurs on tea tender leaves in many tea cultivation regions of Hubei, China, was characterized and distinguished from other known diseases in this study. The causal agents were identified to be closely associated with three Pestalotiopsis species. According to the morphological characteristics and phylogenetic relationships, the main pathogenic species was identified as $P$. theae. Our results provide diagnostic tools that should help improve the prevention and management of this economically important disease.

\section{Acknowledgments}

This work was supported by grants from the National Science Foundation of China (31071662 and 30800746) and Natural Science Foundation of Hubei Province (2014CFB315). The authors declare no competing interests. The authors thank Professor Dejiang Ni, College of Horticulture and Forestry Sciences, Huazhong Agricultural University, Wuhan, Hubei 430070, China, for kind help in sample collection and partial financial support, and Associate Professor Jiankun Zhang, College of Science, Wuhan University of Technology, Wuhan, Hubei 430070, China, for kind help in preparing English.

\section{Literature Cited}

Bai, Q., Zhai, L. F., Chen, X. R., Hong, N., Xu, W. X., and Wang, G. P. 2015. Biological and molecular characterization of five Phomopsis species associated with pear shoot canker in China. Plant Dis. 99:1704-1712.

Bhatt, P. R., Pandya, K. B., and Sheth, N. R. 2010. Camellia sinensis (L): The medicinal beverage: A review. Int. J. Pharm. Sci. Rev. Res. 3:6-9.

Bond, T. J. 2012. The origins of tea, coffee and cocoa as beverages. Pages 1-24 in: Teas, Cocoa and Coffee: Plant Secondary Metabolites and Health, 1st Ed. A Crozier, H. Ashihara, and F. T. Barberán, eds. Blackwell Publishing Ltd, Oxford.

Carbone, I., and Kohn, L. M. 1999. A method for designing primer sets for speciation studies in filamentous ascomycetes. Mycologia 91:553-556.

Chen, Y., Wang, W. X., Zhang, A. F., Yang, X., and Xu, Y.-L. 2013. First report of Pestalotiopsis theae on Loquat (Eriobotrya japonica) in Anhui province of China. Plant Dis. 97:558.

Chen, Y. J., Tong, H. R., Wei, X., and Yuan, L. Y. 2016. First report of brown blight disease on Camellia sinensis caused by Colletotrichum acutatum in China. Plant Dis. 100:227.

Chen, Z. M., and Sun, X. L. 2013. Concise identity handbook of major plant diseases and insect pests of tea, 1st Ed. China Agriculture Press, Beijing, China

Freeman, S., Katan, T., and Shabi, E. 1996. Characterization of Colletotrichum gloeosporioides isolates from avocado and almond fruits with molecular and pathogenicity tests. Appl. Environ. Microbiol. 62:1014-1020.

Ge, Q. X., Chen, Y. X., and Xu, T. 2009. Fungi of China Local Records: Genus Pestalotiopsis. 1st ed. China Science Publishing, Beijing, China.

Glass, N. L., and Donaldson, G. C. 1995. Development of primer sets designed for use with the PCR to amplify conserved genes from filamentous ascomycetes. Appl. Environ. Microbiol. 61:1323-1330.

Guba, E. F. 1961. Monograph of Monochaetia and Pestalotia. Harvard University Press, Cambridge, MA.

Guo, M., Pan, Y. M., Dai, Y. L., and Gao, Z. M. 2014. First report of brown blight disease caused by Colletotrichum gloeosporioides on Camellia sinensis in Anhui province, China. Plant Dis. 98:284.

Jeewon, R., Liew, E. C., Simpson, J. A., Hodgkiss, I. J., and Hyde, K. D. 2003. Phylogenetic significance of morphological characters in the taxonomy of Pestalotiopsis species. Mol. Phylogenet. Evol. 27:372-383.

Ma, J.-Q., Yao, M.-Z., Ma, C.-L., Wang, X.-C., Jin, J.-Q., Wang, X.-M., and Chen, L. 2014. Construction of a SSR-based genetic map and identification of QTLs for catechins content in tea plant (Camellia sinensis). PLoS One 9:e93131.

Maharachchikumbura, S. S. N., Hyde, K. D., Grenewald, J. Z., and Crous, P. W 2014. Pestalotiopsis revisited. Stud. Mycol. 79:121-186.

Mahmood, T., Akhtar, N., and Ali Barkat, K. 2010. The morphology, characteristics and medicinal properties of Camellia sinensis' tea. J. Med. Plants Res. 4: 2028-2033.

Namita, P., Mukesh, R., and Vijay, K. J. 2012. Camellia sinensis (green Tea): a review. Glob. J. Pharmacol. 6:52-59.

Nicholas, K. B., Nicholas, H. B. J., and Deerfield, D. W. I. 1997. GeneDoc: analysis and visualization of genetic variation. Embnew News 4:14.

O'Donnell, K., and Cigelnik, E. 1997. Two divergent intragenomic rDNA ITS2 types within a monophyletic lineage of the fungus Fusarium are nonorthologous. Mol. Phyl. Evol. 7:103-116.

O’Donnell, K., Kistler, H. C., Cigelnik, E., and Ploetz, R. C. 1998. Multiple evolutionary origins of the fungus causing Panama disease of banana: concordant evidence from nuclear and mitochondrial gene genealogies. Proc. Natl. Acad. Sci. USA 95:2044-2049.

Tamura, K., Peterson, D., Peterson, N., Stecher, G., Nei, M., and Kumar, S. 2011 MEGA5: molecular evolutionary genetics analysis using maximum likelihood, evolutionary distance, and maximum parsimony methods. Mol. Biol. Evol. 28: 2731-2739. 
Tuset, J. J., Hinarejos, C., and Mira, J. L. 1999. First report of leaf blight on sweet persimmon tree by Pestalotiopsis theae in Spain. Plant Dis. 83:1070.

Wang, L., Wang, Y., Cao, H., Hao, X., Zeng, J., Yang, Y., and Wang, X. 2016. Transcriptome analysis of an anthracnose-resistant tea plant cultivar reveals genes associated with resistance to Colletotrichum camelliae. PLoS One 11:e0148535.

White, T. J., Bruns, T., Lee, S., and Taylor, J. W. 1990. Analysis of phylogenetic relationships by amplification and direct sequencing of ribosomal RNA genes. Pages 315-322 in: PCR Protocols: A Guide to Methods and Applications. M. A. Innis, D. N. Gelfand, J. J. Sninsky, and T. J. White, eds. Academic Press, San Diego, CA.
Yaegashi, H., Kanematsu, S., and Ito, T. 2012. Molecular characterization of a new hypovirus infecting a phytopathogenic fungus, Valsa ceratosperma. Virus Res. $165: 143-150$

Yang, X. L., Zhang, J. Z., and Luo, D. Q. 2012. The taxonomy, biology and chemistry of the fungal Pestalotiopsis genus. Nat. Prod. Rep. 29:622-641.

Zhang, Y. M., Maharachchikumbura, S. S. N., Wei, J. G., McKenzie, E. H. C., and Hyde, K. D. 2012. Pestalotiopsis camelliae, a new species associated with grey blight of Camellia japonica in China. Sydowia 64:335-344.

Zhou, L. X., and Xu, W. X. 2014. First report of Alternaria alternata causing leaf spots of tea (Camellia sinensis) in China. Plant Dis. 98:697. 